Bol. Soc. Bot. México 46:75-88 (1984).

\title{
La vegetación acuática vascular de seis lagos-cráter del estado de Puebla, México ${ }^{1}$
}

\author{
Pedro Ramírez-García y Alejandro Novelo R. ${ }^{2}$
}

\begin{abstract}
RESUMEN. Las aguas de los seis lagos presentan una elevada concentración de sales y fueron divididos en dos grupos de acuerdo a la concentración de las mismas: el primero, denominado "concentrados" o "salinos", formado por los lagos Alchichica y Atexcac y el segundo, denominado "diluidos", formado por los cuatro lagos restantes. La flora acuática está representada por catorce especies de angiospermas, pertenecientes a trece géneros y nueve familias. La poca diversidad de especies presentes en estos lagos se debe principalmente a las altas concentraciones iónicas del agua y secundariamente al pobre contenido de nutrientes en los sedimentos. La distribución de las especies en los lagos está influida fundamentalmente por los fuertes vientos comúnmente secos y fríos que dominan en esta región y a las pronunciadas pendientes que rápidamente se sumergen a profundidad.
\end{abstract}

ABSTRACT. These lakes were divided into two groups according to their salt concentrations: The Alchichica and Atexcac lakes are "concentrated" or "saline", and the remaining four lakes are "dilute". The aquatic flora includes 9 families, 13 genera and 14 species of angiosperms. The low species diversity is due primarily to the high concentration of salts, and, secondarily, to the low nutrient content of the sediments. The distribution of the species in the lakes is fundamentally influenced by the strong cold and dry winds in the area, and the pronounced steep slopes of the lake bottoms.

\section{INTRODUCCIÓN}

El estudio de la vegetación acuática vascular en nuestro país aún se encuentra en sus etapas iniciales de desarrollo, debido al hábitat particular en el cual se desarrollan estas plantas y a la metodología tan particular que se necesita para llevar a cabo una buena colecta florística. En el caso de los lagos-cráter, las dificultades aumentan por la inaccesibilidad de algunos cuerpos de agua o por las condiciones ambientales adversas dadas por las bajas temperaturas de sus aguas y la profundidad que llegan a tener.

A pesar de que nuestro país puede considerarse como eminentemente volcánico y

${ }^{1}$ Este trabajo representa una parte de la tesis de licenciatura presentada por P. Ramírez-García en la Escuela Nacional de Estudios Profesionales, Plantel Zaragoza, México.

2 Departamento de Botánica, Instituto de Biología, Universidad Nacional Autónoma de México. Apdo. Postal 70-233, 04510 México, D.F.

Ramírez-Gracía P, Novelo-R A. 1984. La vegetación acuática vascular de seis lagos-cráter del estado de Puebla, México. Boletín de la Sociedad Botánica de México 46: 75-88. 
que los lagos-cráter están bien representados (p. ej. los del Valle de Santiago y el lago de Tacámbaro en Guanajuato, las calderas del Valle de México, los lagos-cráter del estado de Puebla, el del volcán Popocatépetl y los del Sol y la Luna del Nevado de Toluca), hasta el momento, de ninguno de ellos se conoce su flora con exactitud.

Con el objeto de avanzar en el conocimiento de la flora acuática vascular en estos ecosistemas particulares, se hizo el presente estudio en seis lagos-cráter, ubicados en el estado de Puebla.

\section{ANTECEDENTES}

La información más abundante que se tiene de los lagos-cráter de nuestro país se concentra principalmente en los del área escogida, con trabajos como los de Ordóñez (1906), donde describe el origen y constitución de estos lagos; Taylor (1943), De Buen (1945) y Álvarez (1950), que trabajan algunos aspectos faunísticos; Fuentes (1972), quien habla de ellos cuando define las diferentes regiones del estado; Arredondo et al. (1983), quienes describen la batimetría y morfometría, y finalmente Gasca (1982), quien trabajó sobre la génesis de estos lagos.

\section{LOCALIZACIÓN Y DESCRIPCIÓN DE LOS LAGOS}

Los lagos-cráter se localizan en la zona E del estado de Puebla, en dos planicies conocidas como Llanos de San Juan y Llanos de San Andrés. Los primeros se asientan en una planicie de origen lacustre que ocupa la parte $\mathrm{N}$ de la meseta poblana; estos llanos son en parte áridos con afloraciones salinas de tequesquite, formados por material de relleno del Pleistoceno y Reciente. Los Llanos de San Andrés se localizan al pie de los contrafuertes occidentales del Pico de Orizaba, en una amplia superficie arenosa donde predominan depósitos volcánicos recientes y se caracterizan por la notable depresión volcánica lacustre que forman (Ordóñez, 1906).

En los Llanos de San Juan se localizan los lagos Alchichica, Atexcac, La Preciosa y Quechulac y en los Llanos de San Andrés se localizan los lagos Aljojuca y Tecuitlapa (fig. 1). Estos lagos están entre las coordenadas $19^{\circ} 05^{\prime}$ y $19^{\circ} 24^{\prime} \mathrm{N}$ y $97^{\circ} 21^{\prime}$ y $97^{\circ} 34^{\prime} \mathrm{W}$, a altitudes de 2300 y $2510 \mathrm{~m}$.

El clima de estos llanos es muy variable y está influido notablemente por la altitud, la exposición de los vientos secos y la sombra eólica que producen los macizos montañosos que los rodean. Gran parte de los Llanos de San Juan presentan un clima templado seco, con verano seco y poca oscilación térmica $\mathrm{BS}_{1} \mathrm{w}^{\prime \prime} \mathrm{k}$ ' (i’)g, mientras que hacia la parte oriental de estos llanos y los de San Andrés el clima es más bien templado subhúmedo, con lluvias en verano $\mathrm{C}\left(\mathrm{w}_{1}\right)$ big.

En la tabla 1 se resumen algunas de las características morfométricas de estos lagos, donde se aprecia que uno de los lagos más grandes en cuanto al área superficial y al volumen es Alchichica y el más pequeño es el lago Tecuitlapa. En relación con su área superficial y su profundidad, casi todos los lagos, excepto el Tecuitlapa, presentan playas con pendientes muy pronunciadas y la forma de la cuenca es cónica. Las paredes que rodean a estos lagos son altas y en su mayoría están constituidas de tobas basálticas grises, aglomeraciones de lapilli con grandes bombas de basalto, andesitas, porfiritas y en algunos lagos aglomeraciones de tezontle, como en Tecuitlapa. 


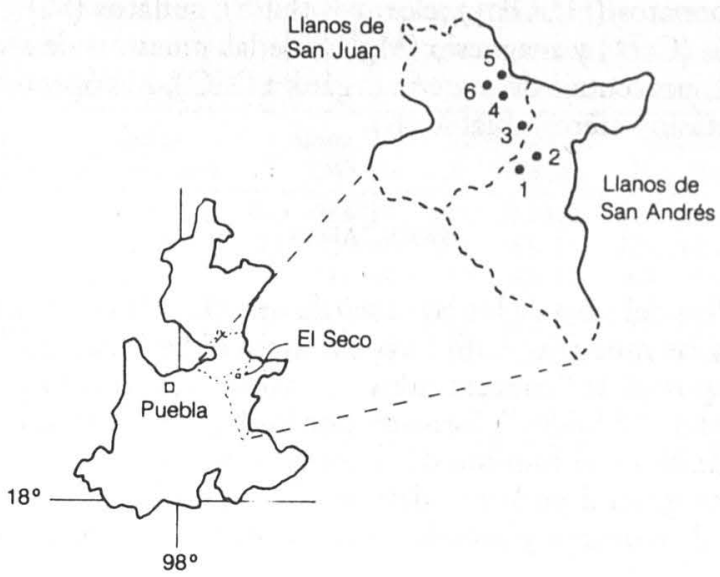

Fig. 1. Localización de los lagos-cráter en el estado de Puebla. 1. Tecuitlapa; 2. Aljojuca; 3. Atexcac; 4. La Preciosa; 5. Quechulac; 6. Alchichica.

\section{Metodología}

Se hicieron doce visitas mensuales con una duración de seis días cada una, a partir de junio de 1980. En estas visitas se hicieron colectas de material botánico, mismas que se encuentran depositadas en el Herbario Nacional del Instituto de Biología de la Universidad Nacional Autónoma de México (MEXU). Para conocer la distribución de las especies en cada uno de los lagos, se hicieron mapas con la ayuda de fotografías aéreas a escala 1:25000; los perfiles y mapas de vegetación están basados en las mediciones hechas en el campo del largo, ancho y altura de las poblaciones, así como la profundidad a la que se encontraban.

Para complementar el estudio, se tomaron muestras bimestrales de agua a nivel de la zona litoral y muestras estacionales de sedimento en cada una de las poblaciones de hidrófitos más importantes. Estas muestras se analizaron para determinar las características físicas y químicas más relevantes de estos ecosistemas lacustres.

Los análisis físicos y químicos aplicados a las muestras de agua fueron: $\mathrm{pH}$, carbona-

TABLA 1. Características morfométricas generales de los lagos-cráter de Puebla*

\begin{tabular}{lcccccc}
\hline & Alchichica & La Preciosa & Quechulac & Aljojuca & Atexcac & Tecuitlapa \\
\hline & & & & & & \\
Área & & & & & & \\
$\quad$ superficial $\left(\mathrm{km}^{2}\right)$ & 1.81 & 0.78 & 0.5 & 0.44 & 0.29 & 0.26 \\
$\quad$ Volumen $\left(\mathrm{m}^{3} \times 10^{6}\right)$ & 69.9 & 16.2 & 10.9 & 11.6 & 6.1 & 0.35 \\
$\begin{array}{l}\text { Profundidad } \\
\text { máxima (m) }\end{array}$ & 64.6 & 45.5 & 40.0 & 50.6 & 39.1 & 2.5 \\
\hline
\end{tabular}

* Datos tomados de Arredondo et al. (1983). 
tos $\left(\mathrm{CO}^{2-}{ }_{3}\right)$, bicarbonatos $\left(\mathrm{HCO}^{1-}{ }_{3}\right)$, cloruros $\left(\mathrm{Cl}^{1-}\right)$, sulfatos $\left(\mathrm{SO}^{2-}{ }_{4}\right)$, sodio $\left(\mathrm{Na}^{1+}\right)$, potasio $\left(\mathrm{K}^{1+}\right)$, calcio $\left(\mathrm{Ca}^{2+}\right)$ y magnesio $\left(\mathrm{Mg}^{2+}\right)$; de las muestras de sedimento se obtuvieron: textura, $\mathrm{pH}$, porcentaje de materia orgánica $(\mathrm{MO})$, nitrógeno total $(\mathrm{N})$, fósforo asimilable $(\mathrm{P})$ y potasio intercambiable $(\mathrm{K})$.

\section{RESUltados}

Aguas. El análisis del agua de los lagos señala una elevada concentración de aniones y cationes en todas las muestras. Con base en estas concentraciones, podemos dividir los lagos en dos grupos: el de "concentrados" o "salinos", formado por los lagos Alchichica y Atexcac, y el de "diluidos", formado por los lagos restantes. Los valores promedio, así como el máximo y el mínimo de la concentración de los iones, se registran en el tabla 2. En forma general podemos definir al agua de los lagos como alcalina, con alta concentración de cloruros y bicarbonatos de sodio y de magnesio.

Sedimentos. El sedimento de todos los lagos es de textura arenosa, con $\mathrm{pH}$ alcalino y baja concentración de nutrientes; los valores promedio se presentan en la tabla 3. Las variaciones de estos factores físicos y químicos del sedimento, no fueron muy importantes entre las diferentes formas de vida de las plantas acuáticas.

Flora y vegetación. La flora acuática encontrada en estos lagos-cráter del estado de Puebla está representada por catorce especies de angiospermas, pertenecientes a trece géneros y nueve familias (tabla 4).

La diversidad florística fue mayor en aquellos lagos descritos anteriormente como "diluidos", encontrándose un máximo de siete especies en el lago Tecuitlapa. Por el contrario, la menor diversidad se encontró en los lagos denominados "concentrados", entre los que destaca Alchichica, el cual solamente estuvo habitado por dos especies.

Casi la mitad de la flora encontrada corresponde a especies que solamente habitan un solo lago, entre las que destacan: Phragmites australis, Sporobolus indicus, Juncus mexi-

TABLA 2. Promedios y rangos de concentración de iones disueltos en el agua de los lagos-cráter, expresados en miliequivalentes por litro $(\mathrm{mEq} / \mathrm{l})$ y salinidad ( $\%$ oo) en partes por mil $(\mathrm{gr} / \mathrm{kg})$

\begin{tabular}{|c|c|c|c|c|c|c|c|c|c|c|c|c|}
\hline & \multicolumn{2}{|c|}{ Alchichica } & \multicolumn{2}{|c|}{ Aljojuca } & \multicolumn{2}{|c|}{ Atexcac } & \multicolumn{2}{|c|}{ La Preciosa } & \multicolumn{2}{|c|}{ Quechulac } & \multicolumn{2}{|c|}{ Tecuitlapa } \\
\hline & $\bar{X}$ & Máx-Min & $\bar{X}$ & Máx-Min & $\bar{X}$ & Máx-Min & $\bar{X}$ & Máx-Mín & $\bar{X}$ & Máx-Mín & $\bar{X}$ & Máx-Mín \\
\hline Cloro & 159.5 & $288.0-82.5$ & 3.6 & $7.0-2.0$ & 179.5 & $330.0-89.0$ & 15.0 & $31.0-5.0$ & 6.7 & $17.0-2.5$ & 3.4 & $6.0-1.5$ \\
\hline Carbonatos & 13.0 & $24.0-3.8$ & 6.6 & $22.0-1.8$ & 6.0 & 8.0- 3.8 & 2.9 & $6.0-0.2$ & 1.3 & $2.0-0.6$ & 4.0 & $8.0-2.6$ \\
\hline Sulfatos & 20.1 & $43.7-10.2$ & 6.2 & $12.5-1.0$ & 5.6 & $12.5-1.0$ & 9.3 & $20.8-2.0$ & 1.7 & $6.2-0.1$ & 0.2 & $0.6-0.1$ \\
\hline Bicarbonatos & 27.5 & $31.8-23.6$ & 12.4 & $23.2-8.0$ & 22.2 & $24.8 \cdot 20.0$ & 8.7 & $12.2-5.2$ & 7.4 & $11.0-4.0$ & 12.1 & $14.8-8.0$ \\
\hline Sodio & 49.5 & $116.0-21.7$ & 12.8 & $22.3-4.0$ & 42.9 & $91.0-21.4$ & 17.2 & $36.0-4.6$ & 10.7 & $19.0-3.7$ & 16.5 & $29.4-4.6$ \\
\hline Magnesio & 32.7 & $39.0-26.6$ & 5.0 & $7.2-3.8$ & 37.0 & $47.5-21.7$ & 13.5 & $17.3-7.5$ & 6.8 & $18.6-3.8$ & 4.5 & $5.3-2.3$ \\
\hline Potasio & 13.8 & $24.1-5.6$ & 1.8 & $3.2-0.7$ & 6.1 & 7.4- 5.1 & 1.4 & $2.3-0.3$ & 0.8 & $1.1-0.4$ & 2.7 & $4.4-1.3$ \\
\hline Calcio & 0.3 & $0.7 \cdot 0.0$ & 1.4 & $1.8-0.7$ & 1.0 & $4.0-0.0$ & 0.5 & $0.7-0.3$ & 2.0 & $8.0-0.4$ & 0.7 & $1.5-0.0$ \\
\hline $\mathrm{pH}$ & 9.0 & $9.1-8.7$ & 8.4 & $9.0-7.5$ & 8.3 & $8.6-7.3$ & 8.4 & $8.9-8.0$ & 8.3 & $8.9-7.5$ & 8.5 & $9.5-7.8$ \\
\hline $\begin{array}{l}\text { Salınidad } \\
\% \text { oo }\end{array}$ & 10.5 & $18.1-5.2$ & 0.2 & $0.4-0.1$ & 11.3 & $20.8-5.6$ & 0.9 & $1.9-0.3$ & 0.4 & $1.0-0.1$ & 0.2 & $0.3-0.09$ \\
\hline
\end{tabular}


TABLA 3. Promedios y rangos de concentración de arenas, limos, $\mathrm{pH}$, materia orgánica y nitrógeno total, así como partes por millón de fósforo asimilable y miliequivalentes por $100 \mathrm{~g}$ de suelo de potasio intercambiable en sedimentos

\begin{tabular}{|c|c|c|c|c|c|c|c|c|c|c|c|c|}
\hline & \multicolumn{2}{|c|}{ Alchichica } & \multicolumn{2}{|c|}{ Aljojuca } & \multicolumn{2}{|c|}{ Atexcac } & \multicolumn{2}{|c|}{ La Preciosa } & \multicolumn{2}{|c|}{ Quechulac } & \multicolumn{2}{|c|}{ Tecuitlapa } \\
\hline & $\bar{X}$ & Máx-Min & $\bar{X}$ & Máx-Min & $\bar{X}$ & Máx-Min & $\bar{X}$ & Máx-Min & $X^{r}$ & Máx-Min & $\bar{X}$ & Máx-Min \\
\hline Arenas & 80.5 & $91.6-66.6$ & 87.2 & $95.0-76.6$ & 85.2 & $95.4-78.4$ & 64.9 & $85.0-35.0$ & 75.6 & $88.4-35.0$ & 89.7 & $96.6-80.0$ \\
\hline Limos & 18.4 & 31.8- & 11.2 & 21.8 & 13.3 & 20.0 & 26.5 & 36.8 & 22.5 & & 8.1 & 1.8 \\
\hline $\mathrm{pH}$ & 7.3 & $7.6-6.9$ & 8.2 & $8.7 \cdot 7.5$ & 7.3 & $8.7-6.6$ & 6.9 & $8.1-6.2$ & 6.9 & 7.6- 6.5 & 8.2 & $9.2-6.8$ \\
\hline & 4.7 & & 2.9 & $6.2-1.28$ & 2.7 & & 5.6 & $10.5-0.69$ & 3.5 & & 1.5 & $2.5-0.69$ \\
\hline Nitrógeno & 0.25 & $0.6-0.0$ & 0.24 & $0.4-0.11$ & 0.19 & $0.4-0.08$ & 0.4 & & 0.3 & -0.11 & 0.14 & $0.3-0.01$ \\
\hline Fósfor & 0.23 & $0.7-$ & 8.4 & $12.6-4.2$ & 5.3 & $13.3-0.0$ & 1.0 & & 6.8 & $15.4-0.0$ & 17.3 & $28.0-8.4$ \\
\hline Potasio & 1.3 & $2.0-0.9$ & 1.1 & $1.7-0.7$ & 1.2 & 2.1- 0.8 & 1.1 & $1.7-0.9$ & 1.3 & 2.3- 0.8 & 1.5 & 1.9- 1.3 \\
\hline
\end{tabular}

canus, Lemna gibba, Typha domingensis y Lilaeopsis schaffneriana. Las siete especies restantes tienen una distribución un poco mayor en esta región, ya que habitan dos o tres lagos. Potamogeton pectinatus fue el hidrófito más común, puesto que se colectó en cinco lagos y se han visto ejemplares de herbario que atestiguan su presencia en el sexto.

La vegetación acuática estudiada está caracterizada por tres formas de vida, en orden decreciente de importancia: los hidrófitos enraizados emergentes, los enraizados sumergidos y los libremente flotadores (tabla 4).

Once especies representan a los hidrófitos enraizados emergentes, entre los que destacan por su distribución y abundancia: Scirpus californicus, Juncus andicola, Eleocharis montevidensis, Phragmites australis y Typha domingensis. Los hidrófitos enraizados sumergidos solamente están representados por dos especies: Potamogeton pectinatus y Ruppia maritima. La única especie representante de los hidrófitos libremente flotadores fue Lemna gibba.

Lago Tecuitlapa. Las especies más importantes por su distribución y abundancia fueron E. montevidensis y J. andicola. La primera se encontró distribuida en forma densa y regular alrededor de casi todo el lago, formando una franja de aproximadamente dos metros de ancho que ocupó la zona litoral y supralitoral. En algunos lugares $E$. montevidensis era sustituida por Juncus andicola que se presentó en forma agregada, formando manchones dispersos alrededor del lago y también bordeando la pequeña isla que se encuentra en el centro. Esta especie ocupó principalmente la zona supralitoral y litoral al E del lago. Otra especie fue Juncus mexicanus encontrada solamente en el $\mathrm{N}$ del lago, ocupando la zona supralitoral. Las especies restantes sólo se presentaron durante la época favorable del año, correspondiente al verano y parte del otoño (fig. 2).

Lago Aljojuca. De las cinco especies de hidrófitos encontrados, Potamogeton pectinatus y Typha domingensis fueron los más abundantes. La población de $P$. pectinatus se distribuyó en forma densa y regular, ocupando la zona mesolitoral al SE del lago, formando una franja de $16 \mathrm{~m}$ de ancho y hasta una profundidad de $8 \mathrm{~m}$, mientras que Typha domingensis se presentó en forma agregada en algunas partes del borde $\mathrm{S}$ del lago, ocupando la zona supralitoral y litoral. Las otras especies que se encontraron creciendo, no formaron comunidades importantes, puesto que fueron ramoneadas constantemente por el ganado (fig. 3). 
TABLA 4. Listado florístico de las especies encontradas en los lagos-cráter de Puebla y la forma de vida que presentan

Familia-especie

Cyperaceae

Cyperus leavigatus L. (1)

Eleocharis montevidensis Kunth (1)

Scirpus californicus (Mey.) Steud. (1)

Gramineae

Phragmites australis (Cav.) Trin. ex Steud. (1)

Sporobolus indicus (L.) R. Br. (1)

Juncaceae

Juncus andicola Hook. (1)

J. mexicanus R. \& S. (1)

Lemnaceae

Lemna gibba L. (3)

Potamogetonaceae

Potamogeton pectinatus L. (2)

Ranunculaceae

Ranunculus cymbalaria Pursh. (1)

Ruppiaceae

Ruppia maritima L. (2)

Typhaceae

Typha domingensis Pers. (1)

Umbelliferae

Hydrocotyle verticillata Thunb. (1)

Lilaeopsis schaffneriana (Schlecht.)

C. \& R. (1)

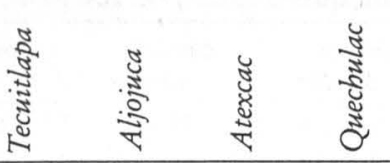

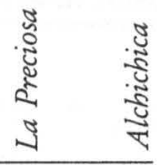

$\begin{array}{ll} & \mathrm{X} \\ \mathrm{X} & \mathrm{X}\end{array}$

X

$\mathrm{X}$
$\mathrm{X}$

$\mathrm{X}$

X X

X

X

$\mathrm{X}$

$\mathrm{X}$

X

$\mathrm{X} \quad \mathrm{X} \quad \mathrm{X} \quad \mathrm{X} \quad \mathrm{X}$

$\mathrm{X} \quad \mathrm{X}$

X

$\mathrm{X}$

(1) Hidrófito enraizado emergente, (2) hidrófito enraizado sumergido, y (3) hidrófito libre flotador.* Especie no colectada durante el estudio, atestiguada por ejemplares de herbario.

Lago Atexcac. En este lago se encontraron cinco especies de hidrófitos. Dos de ellos estuvieron presentes durante todo el año y los restantes sólo durante la estación de invierno y parte de la primavera. La especie Pharagmites australis, presente durante todo el año, se distribuyó en forma agregada en algunas partes de la orilla del lago, ocupando la zona supralitoral y litoral. Otra especie que se presentó durante todo el año fue Cyperus laevigatus, hidrófito emergente que ocupó la zona litoral formando pequeños manchones en 
la playa NW. Potamogeton pectinatus se presentó en forma muy abundante sólo durante los meses de otoño e invierno, ocupando la zona mesolitoral de la playa NE hasta una profundidad de $6 \mathrm{~m}$ (fig. 4). Las otras especies que se presentaron temporalmente en este lago, como Ruppia maritima, sólo lo hicieron en forma muy escasa y durante uno o dos meses de la estación de primavera.

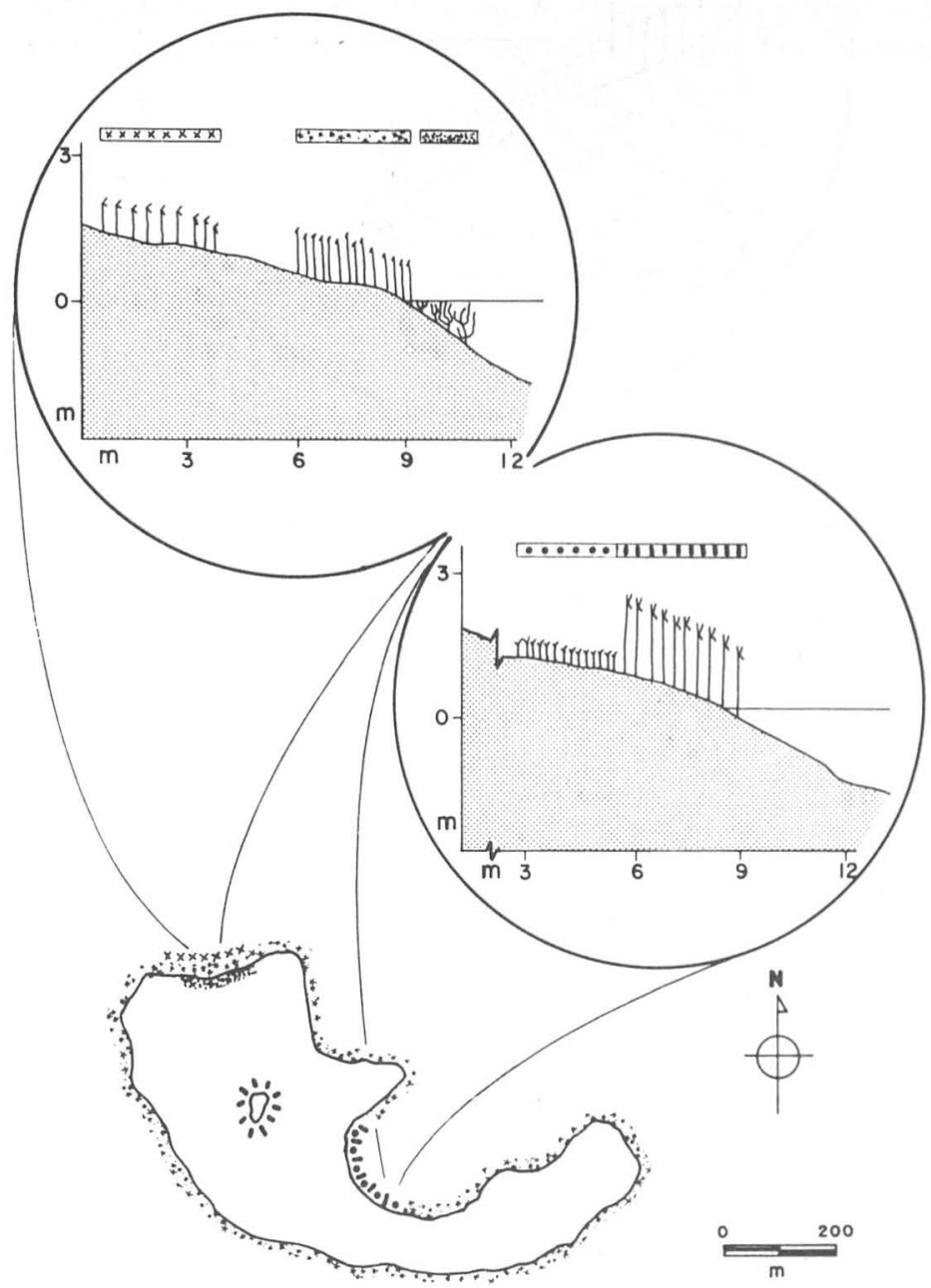

Fig. 2. Distribución de la vegetación acuática en el lago-cráter Tecuitlapa. Clave de símbolos (xxx) Juncus mexi-

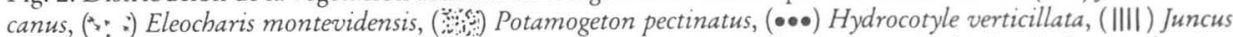
andicola y en las siguientes figuras: ( 4 世) Typha domingensis, (vvv) Phragmites australis, (ooo) Cyperus laevigatus, (///) Scirpus californicus, ('iti? ) Ruppia maritima. 


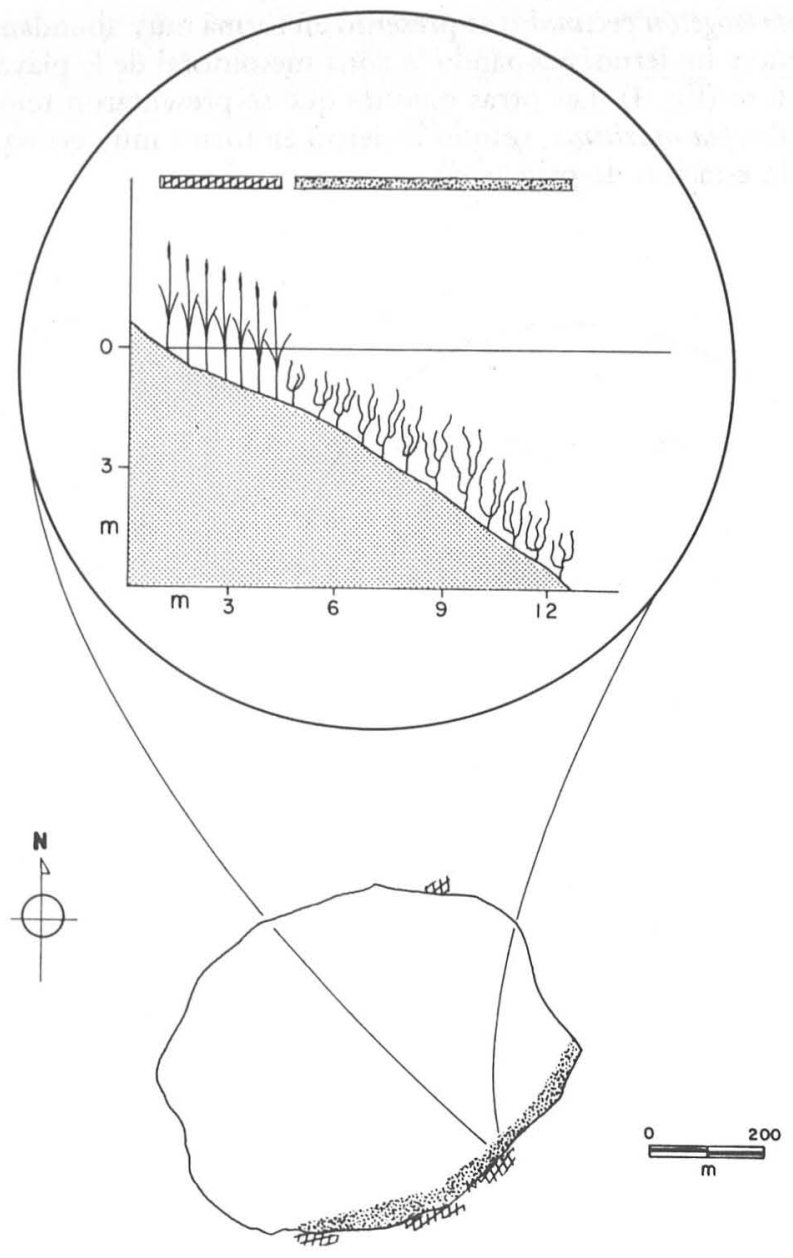

Fig. 3. Distribución de la vegetación acuática en el lago-cráter Aljojuca (ver clave de símbolos, fig. 2).

Lago La Preciosa. En este lago se encontraron cuatro especies de hidrófitos, siendo los más importantes Scirpus californicus, Potamogeton pectinatus y Juncus andicola. El hidrófito enraizado $S$. californicus se encontró distribuido en forma densa y regular al S del lago, ocupando la zona supralitoral y litoral. Potamogeton pectinatus formó una franja densa y regular alrededor del lago, ocupando la zona mesolitoral en algunos lugares hasta $10 \mathrm{~m}$ de profundidad. Por último, J. andicola presentó una distribución muy restringida en forma de una franja densa en un tramo de playa $\mathrm{N}$ ocupando la zona supralitoral (fig. 5).

Lago Quechulac. De las cinco especies de hidrófitos colectados, Scirpus californicus 
y Potamogeton pectinatus fueron los representados en todo el borde del lago a través del año. Scirpus californicus se presentó en forma densa formando una franja de aproximadamente $8 \mathrm{~m}$ de ancho alrededor del lago, ocupando la zona litoral y supralitoral, mientras que $P$. pectinatus también abundó, formando una franja de más de $20 \mathrm{~m}$ de ancho en las partes más someras de la zona mesolitoral, hasta una profundidad de $8 \mathrm{~m}$. Las otras especies de hidrófitos se encontraron en los bordes del lago en forma irregular, inconspicua y temporal (fig. 6).

Lago Alchichica. Dos especies de hidrófitos, Ruppia maritima y Cyperus laevigatus se encontraron durante el estudio; no obstante, existen dos registros de Potamogeton pec-

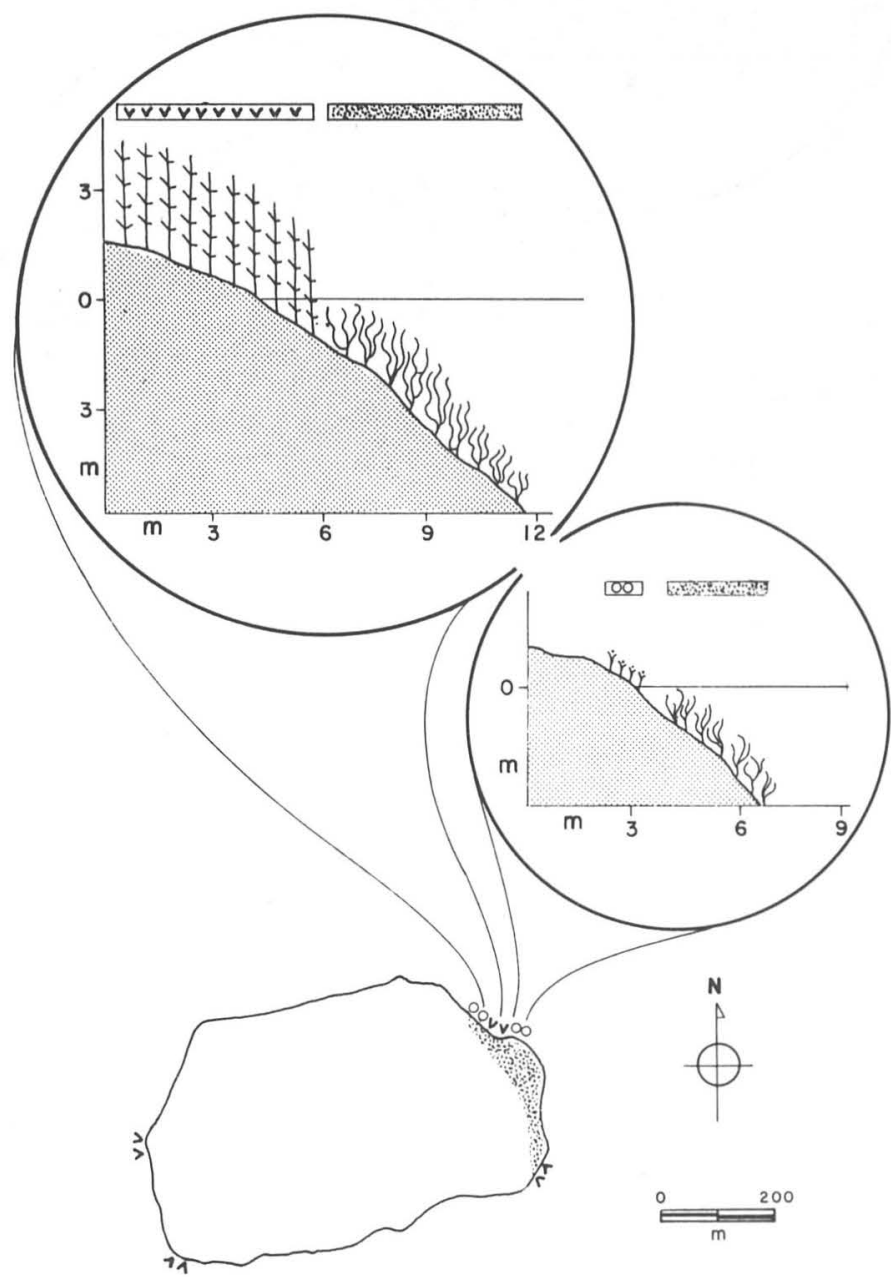

Fig. 4. Distribución de la vegetación acuática en el lago-cráter Atexcac (ver clave de símbolos, fig. 2). 


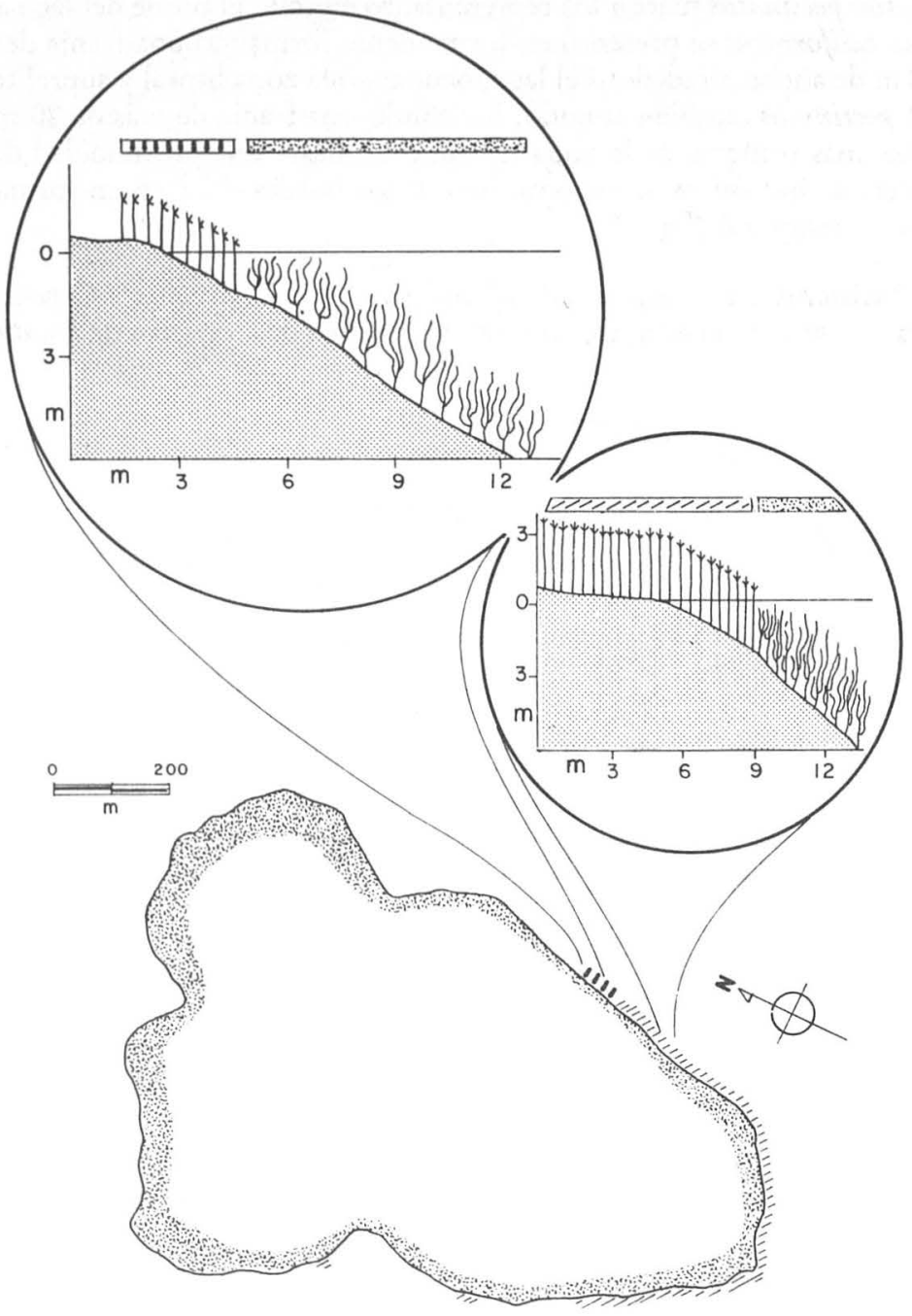

Fig. 5. Distribución de la vegetación acuática en el lago-cráter La Preciosa (ver clave de símbolos, fig. 2).

tinatus para este lago: un ejemplar depositado en el herbario del Missouri Botanical Garden (MO) y otro en el herbario de la Escuela Nacional de Ciencias Biológicas, del Instituto Politécnico Nacional (ENCB). Ruppia maritima fue el hidrófito dominante y se presentó formando una franja de hasta $20 \mathrm{~m}$ de ancho, ocupando la zona litoral en pequeñas caletas de hasta $3 \mathrm{~m}$ de profundidad. La otra especie, C. laevigatus, se localizó en el borde de todo el lago, siendo ramoneada constantemente por el ganado (fig. 7). 


\section{DisCUSIÓN}

La poca diversidad de especies presentes en estos lagos seguramente se debe a la naturaleza tan extrema de los mismos, caracterizada por las altas concentraciones iónicas del agua y secundariamente por el pobre contenido de nutrientes en los sedimentos, mientras que la distribución de las especies en los lagos está influida fundamentalmente por los fuertes vientos comúnmente secos y fríos que dominan en esta región y las pronunciadas pendientes que rápidamente se sumergen a profundidad.

El oleaje que se produce por la acción de los vientos también influye de manera indirecta sobre la distribución de las especies, principalmente de los hidrófitos enraizados emergentes, erosionando el borde en donde estos vegetales se desarrollan e impi-

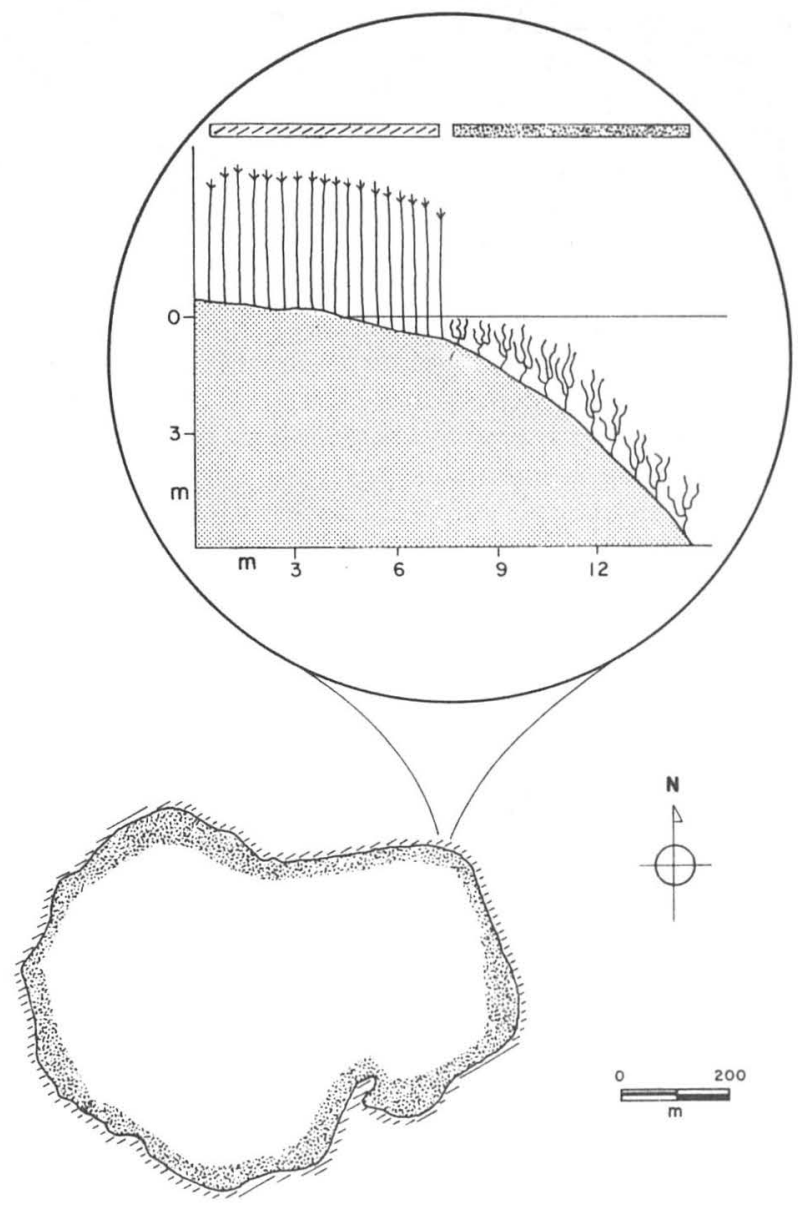

Fig. 6. Distribución de la vegetación acuática en el lago-cráter Quechulac (ver clave de símbolos, fig. 2). 


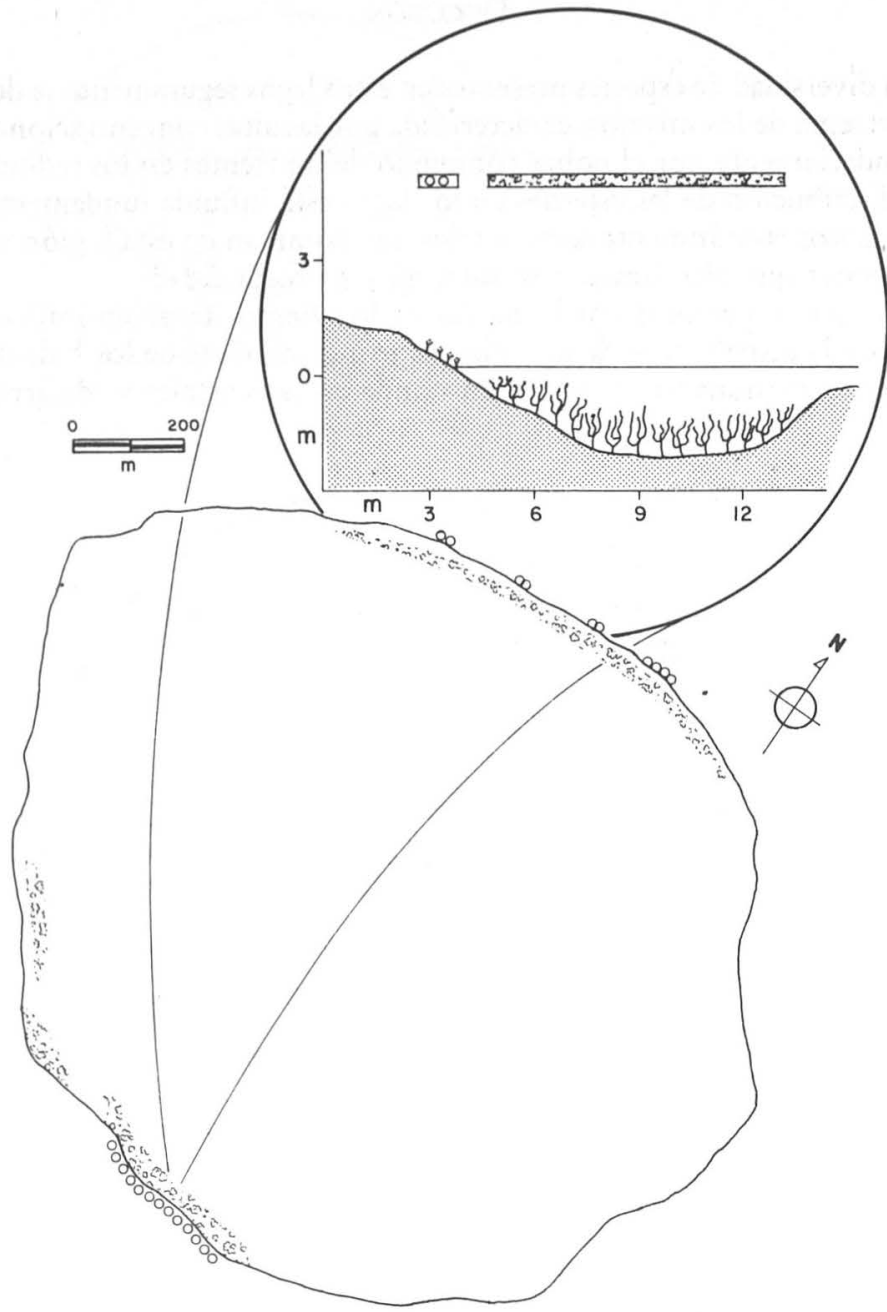

Fig. 7. Distribución de la vegetación acuática en el lago-cráter Alchichica (ver clave de símbolos, fig. 2).

diendo el establecimiento de nuevos individuos, como ha sido sugerido por Hutchinson (1975).

La mayoría de las especies encontradas en estos lagos son indicadoras de esas condiciones extremas en el medio, puesto que un buen número de ellas presentan una distribución amplia a través del mundo y por consiguiente amplia tolerancia tanto a condiciones climáticas como a condiciones físicas y químicas del agua y del sedimento. Como ejemplo podemos citar a Potamogeton pectinatus, Ruppia maritima, Scirpus californicus y Typha domingensis. 
Las únicas especies que pudieron habitar los lagos "concentrados" fueron aquellas que podemos considerar como hidrófitos estrictos, Ruppia maritima y Potamogeton pectinatus. Estas dos especies son consideradas por Ungar (1974) como plantas acuáticas "halofíticas". McMillan (1974) considera a Ruppia como "pasto marino", debido a su amplia tolerancia a la salinidad, ya que se le puede encontrar en ambientes con salinidad nula hasta extrema.

Comúnmente estas dos especies vasculares, junto con algunas algas carofitas, son los únicos elementos vegetales que crecen en los hábitat salinos o salobres tanto de zonas costeras como continentales, como ha sido ya mencionado por Moyle (1945), Penfound (1953), Rzedowski (1957), Hynes (1960), Cole (1963), Ungar (1964, 1965, 1970), Sculthorpe (1967), Ungar et al., (1969), Armitage y Fassett (1971), entre otros.

Con respecto a las especies encontradas en el grupo de lagos "diluidos", muchas de ellas han sido mencionadas por Duncan (1974) como parte de la flora halofítica de la región oriental de Norteamérica y $\mathrm{N}$ de México, entre las que están: Typha domingensis, Ranunculus cymbalaria, Phragmites australis, algunas especies de Lilaeopsis, Juncus y Scirpus y obviamente Ruppia maritima y Potamogeton pectinatus. Aunque la concentración de sales en estos lagos fue menor, seguramente fue suficiente para continuar siendo excluyente con muchas plantas acuáticas, que por el clima pudieron haberse encontrado en estos ecosistemas acuáticos.

Finalmente los cambios paulatinos que se dan dentro del medio acuático, a diferencia del medio terrestre, como son los lentos cambios en la temperatura y la disminución del efecto del viento y del oleaje, permitieron al hidrófito sumergido $P$. pectinatus tener una mayor distribución en los lagos, siempre y cuando la transparencia del agua lo permitiera. En cuanto a los hidrófitos enraizados emergentes, éstos estuvieron más afectados por los fuertes vientos y su presencia o ausencia en alguno de los lagos dependió en gran parte de su resistencia a los vientos. Se observó que, en los bordes de los lagos más cercanos a paredes altas del cráter, los cuales comúnmente estaban más protegidos de los vientos, las comunidades de hidrófitos se desarrollaban en mejor forma. Esto se puede observar en el lago Aljojuca con las poblaciones de T. domingensis, en el lago Atexcac con las poblaciones de Phragmites australis y en el lago La Preciosa con la población de Scirpus californicus.

AgradeCimientos. Queremos agradecer al M.C. Antonio Lot y al Dr. Fernando Chiang, del Instituto de Biología de la Universidad Nacional Autónoma de México, la revisión crítica del manuscrito y al Conacyt por la beca otorgada durante el desarrollo del trabajo y el apoyo parcial a través del Proyecto PCECNAL-790236.

\section{LITERATURA CITADA}

Álvarez, J. 1950. Contribución al conocimiento de los peces de la región de Los Llanos, estado de Puebla (México). An. Esc. Nac. Ci. Biol. México. 69(2):81-107.

Armitage, K.B., y N.C. Fassett. 1971. Aquatic plants of El Salvador. Arch. Hydrobiol. 69(2):234-255.

Arredondo F.J., L. Borrego, R. Castillo y M. Valladolid. 1983. Batimetría y morfometría de los lagos "maars" de la Cuenca de Oriental, Puebla, México. Biótica 8(1):37-47.

ColE, G.A. 1963. The American southwest and Middle America, pp. 393-434. En: D.G. Frey (Ed.), Limnology in North America. University of Wisconsin Press, Madison, Wisconsin.

De BUEN, F. 1945. Investigaciones sobre la ictiología mexicana I. Atherinidạe de aguas continentales de México. An. Inst. Biol. Univ. Nac. Autón. México 16(2):475-532. 
DUNCAN, W.H. 1974. Vascular halophytes of the Atlantic and Gulf coast of North America North of Mexico, pp. 23-50. En: R. Reimold y W. Queen (Eds.), Ecology of halophytes. Academic Press, New York.

Fuentes, A.L. 1972. Regiones naturales del estado de Puebla. Direc. de Publ. Univ. Nal. Autón. México.

GASCA, D.A. 1982. Génesis de los lagos-cráter de la Cuenca de Oriental. Colec. Cient. Inst. Nac. Antropol. Hist. 98, México.

Hutchinson, G.E. 1975. The distribution of macrophytes in lakes, pp. 408-508. En: A treatise on limnology. Vol. III. John Wiley, New York.

Hynes, H.B.N. 1960. The biology of polluted waters. University Press, Liverpool.

MCMilLaN, C. 1974. Salt tolerance of mangroves and submerged aquatic plants, pp. 379-390. En: R. Reimold y W. Queen (Eds.), Ecology of halophytes. Academic Press, New York.

MOYLE, B.J. 1945. Some chemical factors influencing the distribution of aquatic plants in Minnesota. Amer. Midl. Nat. 34:402-420.

Ordónez, E. 1906. Los Xalapascos del estado de Puebla. Paregones Inst. Geol. México 1(8):348-405.

Penfound, W.T. 1953. Plant communities of Oklahoma lakes. Ecology 34:561-583.

Rzedowski, J. 1957. Algunas asociaciones vegetales de los terrenos del Lago de Texcoco. Bol. Soc. Bot. México 21:19-33.

Sculthorpe, C.D. 1967. The biology of aquatic vascular plants. Edward Arnold, Londres.

TAYLOR, E.H. 1943. A new amblystomid salamander adapted to brackish water. Copeia 3:151-156.

UnGar, I.A. 1964. A phytosociological analysis of the Big Salt Marsh, Stafford Country, Kansas. Trans. Kansas Acad. Sci. 67:50-64.

1965. An ecological study of the vegetation of the Big Salt Marsh, Stafford Country, Kansas. Univ. Kansas Sci. Bull. 46:1-98.

1970. Species-soil relationships on sulfate dominated soils in South Dakota. Amer. Midl. Nat. 83:343-357. 1974. Inland halophytes of the United States, pp. 235-305. En: R. Reimold y W. Queen (Eds.). Ecology of halophytes. Academic Press, New York.

W. Hogan y M. McClelland. 1969. Plant communities of saline soils at Lincoln, Nebraska. Amer. Midl. Nat. 82:564-577. 